\author{
Military Technical College \\ Kobry El-Kobbah, \\ Cairo, Egypt.
}

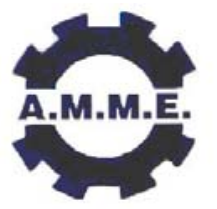

\title{
SOCIAL POTENTIAL MODEL TO SIMULATE EMERGENT BEHAVIOUR FOR SWARM ROBOTS
}

\author{
MABROUK $^{*}$ M. H. and MCINNES ${ }^{* *}$ C.R
}

\begin{abstract}
Swarm robotics has a wide range of applications in numerous fields from space and subsea exploration to the deployment of teams of interacting artificial agents in disposal systems. In this paper, we introduce a model to simulate the emergent behaviour of multiagent robot systems, based on principles from physical mechanics. The model is based on mutual interactions among the swarm individuals. The main elements of these interactions are repulsion forces, attraction forces, alignment forces and dissipative forces generated by the swarm members. Using statistical tools, which are used to investigate simulated group behaviour, we discuss the importance of introducing some dissipation to the system as well as the effect of the interaction parameters on various components of the model.
\end{abstract}

\section{KEY WORDS}

Robotics, Swarm robots, Emergent behaviour.

$\begin{array}{ll}\text { NOMENCLATURE } \\ C_{a i} & \text { Amplitude of attraction potential of the } \mathrm{i}^{\text {th }} \text { agent. } \\ C_{g} & \text { Amplitude of goal constant potential. } \\ C_{o} & \text { Amplitude of alignment force term. } \\ C & \text { Potential amplitude ratio. } \\ C_{r i} & \text { Amplitude of repulsive potential of the } \mathrm{i}^{\text {th }} \text { agent. } \\ \mathrm{F}_{\text {cohesion }}\left(\mathrm{r}_{i}\right) & \text { Cohesion force exerted on the } \mathrm{i}^{\text {th }} \text { agent. } \\ \mathrm{F}_{\text {alignment }}\left(\mathrm{r}_{i}\right) & \text { Alignment force exerted on the } \mathrm{i}^{\text {th }} \text { agent. } \\ \mathrm{F}_{\text {dissipation }}\left(\mathrm{r}_{i}\right) & \text { Dissipation (friction) force exerted on the } \mathrm{i}^{\text {th }} \text { agent. } \\ \mathrm{F}_{\text {goal }}\left(\mathrm{r}_{i}\right) & \text { Goal attractive force exerted on the } \mathrm{i}^{\text {th }} \text { agent. }\end{array}$

\footnotetext{
"PhD research student, Dept. of Mechanical Engineering, University of Strathclyde, Glasgow, UK.

** Professor, Dept. of Mechanical Engineering, University of Strathclyde, Glasgow, UK.
} 


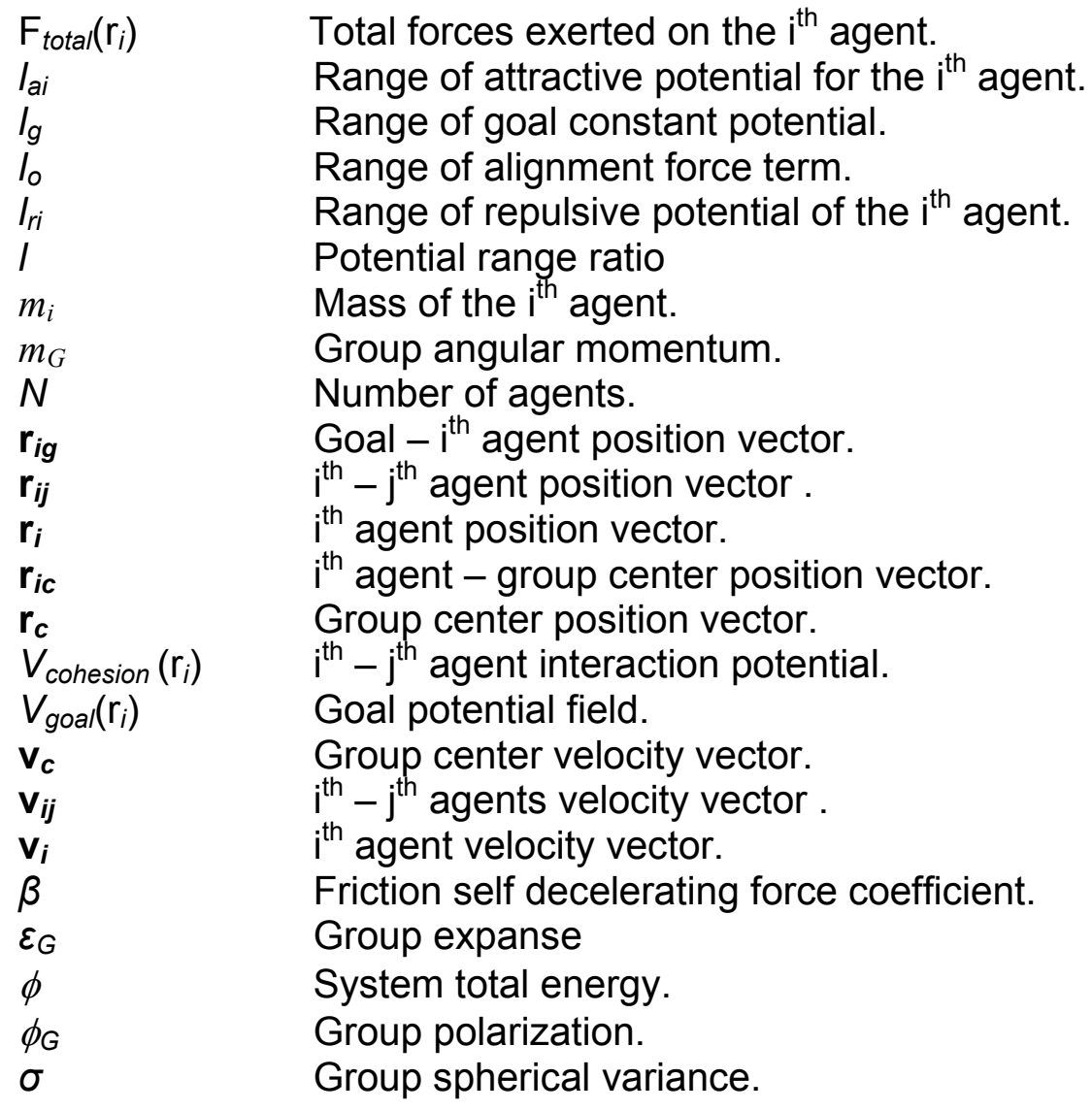

\section{INTRODUCTION}

Swarm robotics is a new and promising approach to the design and control of multi-agent robotic systems. Specific features of aggregations are striking in natural systems whose members have high rates of information exchange such as animal herds, insect swarms, bird flocks, and fish schools [1]. This leads us to discuss one of the most important phenomena in natural systems, which is the emergent behavior.

\section{Emergent Behavior in Literature}

Natural examples of emergent behaviour in groups due to interactions between each of the group's elements are numerous. Beehives, locust swarms, geese flocking, bacterial mutation are all examples of emergent phenomena where a collection of individuals interact without central control to produce results which are not explicitly "programmed". The advantages of emergence-based systems can be summarized in the following: (1) they are robust in a way that the system still works even if one of its unit fails (2) they can adapt to changing parameters environment, which is typical of the real world (3) they don't need to have complete knowledge to achieve a goal (4) they find a reasonable solution quickly and then optimize it. Concerning the simulation of individuals' motion in groups, most simulation studies assume that all individuals are identical. The author in [2] 
discussed that, among a school of fish, the inclusion of a single different fish may alter the group's collective behaviour. Common swarming patterns have been observed and reported in various species in nature [3]. The coherent flock and the single-mill states are among the most common patterns observed in biological swarms [4-5]. The double-mill pattern is also occasionally seen [6]. Models of natural or artificial individuals, which interact through pair-wise long-range attraction and short-range repulsion within a swarm, were introduced [7-12]. In [12], the authors were able to predict stability and emergent patterns of organization starting from the form of the two-body interaction through a model specially designed to simulate the motion of self-accelerated particles. On the other hand, propagating the motion of the swarm using artificial potential fields shows that swarms of interacting particles can relax into vortex-like states [13]. Such evolutionary behaviour may offer new approaches to many classes of information processing problems, which currently prove infeasible. In recent years, there has been much interest in decentralized, multirobot systems due to their potential advantages in many applications over more traditional, monolithic architectures [14]. Another challenge is to design systems that can accomplish their tasks more reliably, faster and cheaper than could be achieved by a single more complex robot. An application is to develop controllers for the individual robots such that the group as a whole performs the desired higher-level task through the coordinated action of the individuals as presented in [15].

Mathematical modeling of emergent behaviors in such systems provides a promising way for more robust designs of multiagent robotic systems. However, before going deep in modeling we need to mention some statistical tools, which are widely used in the field to investigate simulated group movements. These statistics will be used to have insights of our model.

\section{DESCRIPTIVE STATISTICS TO AID MODEL ANALYSIS}

We now consider a system composed of $N$ identical individuals. At a particular time $t$, each individual has an associated position $\mathbf{r}_{i}(t)$ (a column vector of Cartesian coordinates), a velocity vector $\mathbf{v}_{i}(t)$; a mass $m_{i}$ (assuming the individuals' mass is identical and equal to unity for simplicity), where $i=1, \ldots, N$. The group center, which is analogous to the center of mass in a multi-particle physical system, is calculated as the mean of all the individuals' position vectors at time $t$ :

$$
\mathbf{r}_{c}=\frac{1}{N} \sum_{i=1}^{N} \mathbf{r}_{i}
$$

As defined in [16], the group polarization $\left(\phi_{G}\right)$ measures the degree of alignment amongst individuals within the group in a way that a more dispersed group has a lower polarization. The group angular momentum $\left(m_{G}\right)$ is a measure of the degree of rotation of the group around the group center: 
$\phi_{G}=\frac{1}{N}\left|\sum_{i=1}^{N} \mathbf{v}_{i}\right|$

$m_{G}=\frac{1}{N}\left|\sum_{i=1}^{N} \hat{\mathbf{r}}_{i c} \times \mathbf{v}_{i}\right|$

where $|$.$| is the norm of a vector, and \hat{\mathbf{r}}_{i c}$ is the unit relative position vector between the $i^{\text {th }}$ individual and the group center. We can also define the spherical variance as an estimate of the dispersion of the group in the following way [17]:

$\sigma=1-\phi_{G}$

We can then use two measures to compare the relative size of groups; the average nearest neighbor distance (Av. NND) and the group expanse. The nearest neighbor distance, which varies for each individual within the group, is the distance between a particular individual and its closest neighbor [11]. We calculate the average nearest neighbor distance for the group as:

Av. $\mathrm{NND}=\frac{1}{N} \sum_{i=1}^{N} \min _{j \neq i}\left|\mathbf{r}_{i}-\mathbf{r}_{j}\right|$

The group expanse measures the size of the group. It is the average distance between individuals and the group center [11]. Therefore, expanse is calculated as:

$\varepsilon_{G}=\frac{1}{N} \sum_{i=1}^{N} \sqrt{\left(\left|\mathbf{r}_{i}\right|-\left|\mathbf{r}_{c}\right|\right)^{2}}$

An increasing average NND implies that the inter-agent distances are increasing and the group becomes more dispersed while as expanse increases, the physical size of the group increases.

\section{SOCIAL POTENTIAL MODEL FOR SWARM ROBOT EMERGENT BEHAVIOR}

In Ref. [13], a discrete particle model is introduced where the author discussed the emergence of vortex-like behavior that could then be viewed as a constrained minimum energy configuration, which the swarm relaxes into. In [18], we model the collective group behaviour by using a sum of gradient potentials based on exponentially decayed social forces and a linear dissipation force term to prevent agents from abandoning the group due to excessive linear momentum and/or having identical initial positions which result in a violent repulsion of the individuals. This generates behaviour, which is close to that of real biological systems whose members feel a desire to stick together. In this paper we develop the model of cohesive forces to include an alignment force term, which is introduced in [13] to allow individuals to orient their velocities with respect to one another. 
Considering the system defined at the beginning of section 1.2 , the equations of motion will be:

$\mathbf{v}_{i}=d \mathbf{r}_{i} / d t$

$m_{i} \cdot d \mathbf{v}_{i} / d t=\mathbf{F}_{\text {total }}$

Where $\mathbf{F}_{\text {total }}$ is the total force that is exerted on the $\mathrm{i}^{\text {th }}$ agent. It is constituted of cohesion forces among the agents, alignment forces and a dissipation (friction) force.

We now consider only pair-wise interactions among individuals, and we neglect higher order interactions because they are less likely to occur in comparison to the likelihood of pair-wise interactions. We define the attractive and repulsive forces as decaying exponential functions by using the generalized Morse potential [18], giving close to real simulation for the individuals' pair-wise interaction as follows:

$$
V_{\text {cohesion }}\left(\mathbf{r}_{i}\right)=\sum_{j \neq i}^{N} C_{r} e^{-\left|\mathbf{r}_{i}-\mathbf{r}_{j}\right| / l_{r}}-\sum_{j \neq i}^{N} C_{a} e^{-\left|\mathbf{r}_{i}-\mathbf{r}_{j}\right| / l_{a}}
$$

Where the potential is characterized by attractive and repulsive potential fields of strength $C_{a}$ and $C_{r}$ with ranges $I_{a}$ and $I_{r}$ respectively while $C$ is the potential amplitude ratio and $I$ is the potential range ratio $\left(C_{a}, C_{r}, I_{a}\right.$, and $I_{r}$ are positive constants; $C=C_{r} / C_{a}$ and $\left.I=I_{r} / I_{a}\right)$. Now we can derive a physical definition for the social forces acting on the $i^{\text {th }}$ agent, due to the cohesion social potentials. Therefore, it can be seen that:

$$
\mathbf{F}_{\text {cohesion }}\left(\mathbf{r}_{i}\right)=\nabla_{i} V_{\text {cohesion }}\left(\mathbf{r}_{i}\right)=\sum_{j \neq i}^{N}\left[\frac{C_{a}}{l_{a}} e^{-\left|\mathbf{r}_{i}-\mathbf{r}_{j}\right| / l_{a}}-\frac{C_{r}}{l_{r}} e^{-\left|\mathbf{r}_{i}-\mathbf{r}_{j}\right| / l_{r}}\right] \hat{\mathbf{r}}_{i j}
$$

where $\hat{\mathbf{r}}_{i j}$ is the unit vector in the direction of the vector $\mathbf{r}_{i j}$.

We now consider a different perspective inspired from the model introduced in [13], by the addition of an alignment force to the cohesion model. This alignment force, which is designed to act directly on the agents' velocities, allows individuals to orient their velocities with respect to one another. The constant $C_{o}$ is the alignment magnitude, which controls the size of the alignment term, and $I_{0}$ is the range over which the orientation interaction occurs. Therefore, the alignment force will be defined as:

$$
\mathbf{F}_{\text {alignment }}\left(\mathbf{r}_{i}, \mathbf{v}_{i}\right)=-\sum_{j \neq i}^{N} C_{o}\left(\mathbf{v}_{i j}, \hat{\mathbf{r}}_{i j}\right) e^{-\left|\mathbf{r}_{i j}\right| / l_{o}} \hat{\mathbf{r}}_{i j}
$$

Now we use a simple dissipative force, similar to a simple frictional force that directly acts upon each individual. We define the dissipative force acting on the $\mathrm{i}^{\text {th }}$ agent as:

$$
\mathbf{F}_{\text {dissipation }}\left(\mathbf{v}_{i}\right)=-\beta \mathbf{v}_{i}
$$


where $\beta$ is a positive nonzero integer, which governs the amount of dissipation (essentially the coefficient of friction). The total force, which acts upon the $\mathrm{i}^{\text {th }}$ agent at a particular time $t$ according to the model, is therefore defined as:

$\mathbf{F}_{\text {total }}\left(\mathbf{r}_{i}, \mathbf{v}_{i}\right)=\mathbf{F}_{\text {cohesion }}\left(\mathbf{r}_{i}\right)+\mathbf{F}_{\text {alignment }}\left(\mathbf{r}_{i}, \mathbf{v}_{i}\right)+\mathbf{F}_{\text {dissipation }}\left(\mathbf{v}_{i}\right)$

Substituting from Eq. (10-12) in Eq. (13), it can be seen that:

$\mathbf{F}_{\text {total }}\left(\mathbf{r}_{i}, \mathbf{v}_{i}\right)=-\beta \mathbf{v}_{i}+\sum_{j \neq i}^{N}\left(\frac{C_{a}}{l_{a}} e^{-\left|\mathbf{r}_{i}-\mathbf{r}_{j}\right| / l_{a}}-\frac{C_{r}}{l_{r}} e^{-\left|\mathbf{r}_{i}-\mathbf{r}_{j}\right| / l_{r}}-C_{o}\left(\mathbf{v}_{i j}, \hat{\mathbf{r}}_{i j}\right) e^{-\left|\mathbf{r}_{i j}\right| / l_{o}}\right) \hat{\mathbf{r}}_{i j}$

\section{STABILITY ANALYSIS OF THE MODEL}

We will discuss the stability of the model using the Lyapunov stability method. The Lyapunov function used here is the total energy of the system and we will show that the system is stable by proving that the system will slowly leak energy and relax to a minimumenergy state. Substituting from Eq. (10) and Eq. (14) in Eq. (2), and taking the physical definition of force into consideration, it can be concluded that:

$$
m_{i} \dot{\mathbf{v}}_{i}=-\beta \mathbf{v}_{i}-\nabla_{i} V_{\text {cohesion }}\left(\mathbf{r}_{i}\right)-\sum_{j \neq i}^{N} C_{o}\left(\mathbf{v}_{i j}, \hat{\mathbf{r}}_{i j}\right) e^{-\left|\mathbf{r}_{i j}\right| / l_{o}} \hat{\mathbf{r}}_{i j}
$$

The total energy of the system is therefore:

$\phi=\sum_{i=1}^{N} \frac{1}{2} m_{i} \mathbf{v}_{i}^{2}+V_{\text {cohesion }}\left(\mathbf{r}_{i}\right)$

Then, the time derivative of the total energy is given by:

$\dot{\phi}=\sum_{i=1}^{N} \mathbf{v}_{i} \cdot\left(m_{i} \dot{\mathbf{v}}_{i}+\nabla_{i} V_{\text {cohesion }}\left(\mathbf{r}_{i}\right)\right)$

Substituting from Eq. (15) in Eq. (17) it can be seen that:

$$
\dot{\phi}=-\sum_{i=1}^{N} \beta \mathbf{v}_{i}^{2}-\sum_{i=1}^{N} \sum_{j \neq i}^{N} C_{o}\left(\mathbf{v}_{i} \cdot \mathbf{v}_{i j}\right) e^{-\left|\mathbf{r}_{i j}\right| / l_{o}} \hat{\mathbf{r}}_{i j}^{2}
$$

Knowing that $\beta>0, C_{0} \geq 0$, then it can be concluded that $\dot{\phi}<0$, therefore the system is Lyapunov stable, so that the group will slowly leak energy and relax to a minimum-energy state. 


\section{SIMULATION RESULTS AND DISCUSSION}

In this section, we will show the importance of the dissipative force and study the effect of the interaction parameters on the group behaviour. Concerning how the friction coefficient affects the model, we use the model from $\mathrm{Eq} .(7,8,14)$ with default parameters $\left(N_{p}=30, C_{a}=1, C_{r}=1.2, C_{o}=0.1, l_{a}=1, I_{r}=0.2, I_{o}=1.5\right)$ to simulate the individuals' motion. We run our simulations starting from random initial conditions for 500 simulation steps (time enough to reach the minimum-energy state). Logically, the dissipation force only acts to make the individuals slow down and it has nothing to do with the individual's inclination to align with its neighbours. However, the dissipative force has an effect on group polarization; as shown in Fig.1. Therefore, in general, higher dissipation will lead to a lower polarization that indicates a more dispersed group arrangement. Fig. 1 also shows $\beta=0.1$ as corresponding to the workable value of dissipation before a rapid leak of the system's energy. The comparison between the simulation results in Fig.2, without a dissipation term, and Fig.3, with $\beta=0.1$, shows the effect of adding a dissipation term to the system. Clearly, the individuals' velocities are lower for higher dissipation (friction) force.

Also, it is apparent from the comparison between Fig.4 and Fig.5 that the dissipation has a considerable effect on the group angular momentum and the group spherical variance. As the dissipation coefficient increases the speeds of the individuals become lower which cause two effects; first the polarization decreases noticeably, therefore the value of spherical variance approaches unity very soon; secondly the group angular momentum decreases rapidly with time until reaching zero, giving an indication of a faster rate of leak in system energy for higher dissipation.

There are two parameters that control the strength and range of the alignment force respectively, $\left(C_{o}\right)$ and $\left(I_{o}\right)$. Therefore, it is logical to expect that for higher values of $C_{o}$ the group individuals become more likely to emerge in the vortex pattern, which should be particularly affecting the group angular momentum. This is illustrated in Fig.6, where we have altered the alignment magnitude while fixing the values of the other parameters, so that as the value of $C_{o}$ increases the group angular momentum noticeably increases. Also, Fig. 7 shows the effect of the alignment magnitude on polarization. As the value of $C_{o}$ increases, the group polarization decreases (as expected). Logically, the individuals tend to form a less compact cluster for increasing values of $C_{o}$ due to centrifugal action increasing, which has a low effect on the group size. This is shown in Fig.8, which demonstrates that the values of the group expanse and average NND slowly increases as the alignment magnitude increases.

The main effect on the model due to the cohesion forces is caused by the potential ranges $\left(I_{r}\right.$ and $\left.I_{a}\right)$ rather than the potential amplitudes, as these terms are involved in the exponential decay [9]. We expect that the effect of increasing the attraction and repulsion ranges $\left(I_{a}\right.$ and $\left.I_{r}\right)$ are to decrease and increase the size of the group, respectively. The results in Fig.9 show that as the range ratio $I$ increases, which can be due to either $I_{r}$ increasing and/or $l_{a}$ decreasing, the values of group expanse and average NND's increase, indicating a more dispersed group emerges. Also as the range ratio $C$ increases, which can be due to either $\mathrm{C}_{r}$ increasing and/or $\mathrm{C}_{a}$ decreasing, a more dispersed group emerges. 
As shown in Fig.10, when the values of $C$ increase so does the value of group expanse and average NND's.

Until this point, the dissipation force helps to control group behaviour and to make the model suitable for real applications. Now we introduce an additional attraction gradient force that acts on individuals at longer distances than the current repulsion and attraction forces. This attraction force should be an attraction to the group as a whole rather than to individuals. Assuming a potential sink at point $G$ (the goal position for the swarm) where $C_{g}$ is the potential attraction amplitude of the goal and $l_{g}$ is the potential attraction range, then the equation for the goal potential according to generalized Morse potential will be:

$$
V_{\text {goal }}\left(\mathbf{r}_{i}\right)=-C_{g} e^{-\left|\mathbf{r}_{i}-\mathbf{r}_{g}\right| / l_{g}}
$$

Now the total force, Eq. (13), will be modified to include the goal force term:

$$
\mathbf{F}_{\text {total }}\left(\mathbf{r}_{i}, \mathbf{v}_{i}\right)=\mathbf{F}_{\text {repulsion }}\left(\mathbf{r}_{i}\right)+\mathbf{F}_{\text {attraction }}\left(\mathbf{r}_{i}\right)+\mathbf{F}_{\text {alignment }}\left(\mathbf{r}_{i}, \mathbf{v}_{i}\right)+\mathbf{F}_{\text {dissipate }}\left(\mathbf{v}_{i}\right)+\mathbf{F}_{\text {goal }}\left(\mathbf{r}_{i}\right)
$$

Therefore, Eq. (14) will be:

$$
\begin{gathered}
\mathbf{F}_{\text {totalt }}\left(\mathbf{r}_{i}, \mathbf{v}_{i}\right)=\sum_{j \neq i}^{N}\left(\frac{C_{r}}{l_{r}} e^{-\left|\mathbf{r}_{i}-\mathbf{r}_{j}\right| / l_{r}}-\frac{C_{a}}{l_{a}} e^{-\left|\mathbf{r}_{i}-\mathbf{r}_{j}\right| / l_{a}}-C_{o}\left(\mathbf{v}_{i j}, \hat{\mathbf{r}}_{i j}\right) e^{-\left|\mathbf{r}_{i j}\right| / l_{o}}\right) \hat{\mathbf{r}}_{i j} \\
-\beta \mathbf{v}_{i}-\frac{C_{g}}{l_{g}} e^{-\left|\mathbf{r}_{i}-\mathbf{r}_{g}\right| / l_{g}}
\end{gathered}
$$

This form of the model will help in using the model in robot path-planning applications using the artificial potential fields based navigation technique. Fig.11 shows a swarm of agents in a vortex like shape pattern approaching a potential sink at point $G$. Also, as a future work, we can see that the dissipation term will play a vital rule in controlling the agents during navigation in a way that generates swarm emergent behaviour in more efficient and robust navigation techniques.

\section{CONCLUSIONS}

We introduce a model to simulate the emergent behaviour of multi-agent robot systems, based on individuals' mutual interactions, which encounter repulsion forces, attraction forces, alignment forces and dissipative forces among the system's members. Adopting the approach of [9], we discuss the stability of the system by using the Lyapunov stability method to give clear information about the suitability of the model for robot applications. Also by adopting the analysis of some previous work [5] to similar models, the effects of the models' various component parameters on the global behavior of the model are investigated. 
The results show that that when the dissipation coefficient increases, the speeds of the individuals become lower which causes two effects; first the polarization decreases noticeably, therefore the value of spherical variance approaches its maximum value very rapidly; secondly the group angular momentum decreases rapidly with time, giving an indication of a faster rate of leak in system energy for higher dissipation, so that we concluded that $\beta=0.1$ corresponds to the workable value of dissipation before a rapid leak of the system's energy. The results also show that the alignment magnitude affects the group polarization, group angular momentum, and group size. As the value of $C_{o}$ increases, the group polarization decreases (as expected). Also when the value of $C_{o}$ increases the group angular momentum noticeably increases, and then due to centrifugal action, the group expanse and average NND slowly increase. Finally, the results show that that the effects of increasing the attraction and repulsion parameters are to decrease and increase the size of the group, respectively.

\section{REFERENCES}

[1] S. Camazine, J. L. Deneubourg, N. R. Franks, J. Sneyd, G. Theraulaz, and E. Bonabeau, "Self Organization in Biological Systems", Princeton University Press, Princeton, NJ, 2003.

[2] W. L. Romey, "Individual differences make a difference in the trajectories of simulated schools", Ecol. Model, 92: 65-77, 1996.

[3] A. Czirok, E. Ben-Jacob, I. Cohen, and T. Vicsek, "Formation of complex bacterial colonies via self-generated vortices", Phys. Rev. E 54, 1791, 1996.

[4] J K Parrish, L Edelstein-Keshet, "Complexity, Pattern, and Evolutionary Trade-offs in Animal Aggregation”, Science 284:99-101, 1999.

[5] J. K. Parrish, S. V. Viscido and D. Gr"unbaum, "Self organized fish schools: an examination of emergent properties", Biological Bulletin 202, 296-305, 2002.

[6] A. L. Koch, D. White, "The social life style of myxobacteria", Bioessays 201030 $1038,1998$.

[7] J. A. Beecham and K. D. Farnsworth, "Animal group forces resulting from predator avoidance and competition minimization", Journal of Theoretical Biology 198, 533$548,1999$.

[8] H. Levine, W. J. Rappel, and I. Cohen "Self-Organization in Systems of SelfPropelled Agents", Phys. Rev. E, Vol. 63, pp. 017101, 2000.

[9] A. Mogilner, L. Edelstein-Keshet, L. Bent, and A. Spiros, "Mutual interactions, potentials, and individual distance in a social aggregation", Journal of Theoretical Biology 47, 353-389, 2003.

[10] C. M.Topaz, A. L. Bertozzi, and M. A. Lewis, "A nonlocal continuum model for biological aggregation", Bull. Math. Bio.68, 7, 1601 - 1623, 2006.

[11] S. V. Viscido, J. K. Parrish, and D. Gr"unbaum, "The effect of population size and the number of influential neighbours on the emergent properties of fish schools", Ecological Modelling 183, 347-363, 2005.

[12] M. R. D'Orsogna, Y. L. Chuang, A. L. Bertozzi and L. Chayes, "Self-Propelled Agents With Soft-Core Interactions: Patterns, Stability, and Collapse", Phys. Rev. Lett., Vol. 96, 104302, 2006. 
[13] C. Mclnnes, "Vortex formation in swarms of interacting particles", Phys. Rev. E 75, 032904, 2007.

[14] T. Arai, E. Pagello, and L. E. Parker, "Guest editorial: Advances in multirobot systems", IEEE Transactions on Robotics and Automation, 18(5):655-661, 2002.

[15] T. Taylor, P. Ottery and J. Hallam, "Pattern formation for multi-robot applications: Robust, selfrepairing systems inspired by genetic regulatory networks and cellular self-organisation", Technical Report EDI-INFRR- 0971, School of Informatics, University of Edinburgh, 2007.

[16] I. D.Couzin, J. Krause, R. James, G. D. Ruxton, and N. R. Franks, "Collective memory and spatial sorting in animal groups", Journal of Theoretical Biology 218, 111, 2002.

[17] K. Mardia, "Statistics of Directional Data, Probability and Mathematical Statistics", Academic Press, New York, 1972.

[18] M. Mabrouk and C. Mclnnes, "Swarm Potential Fields with Internal Agent States and Collective Behaviour", In Proceeding of Towards Autonomous Robotic Systems (TAROS), pp. 90-96, 2007.

\section{Figures}

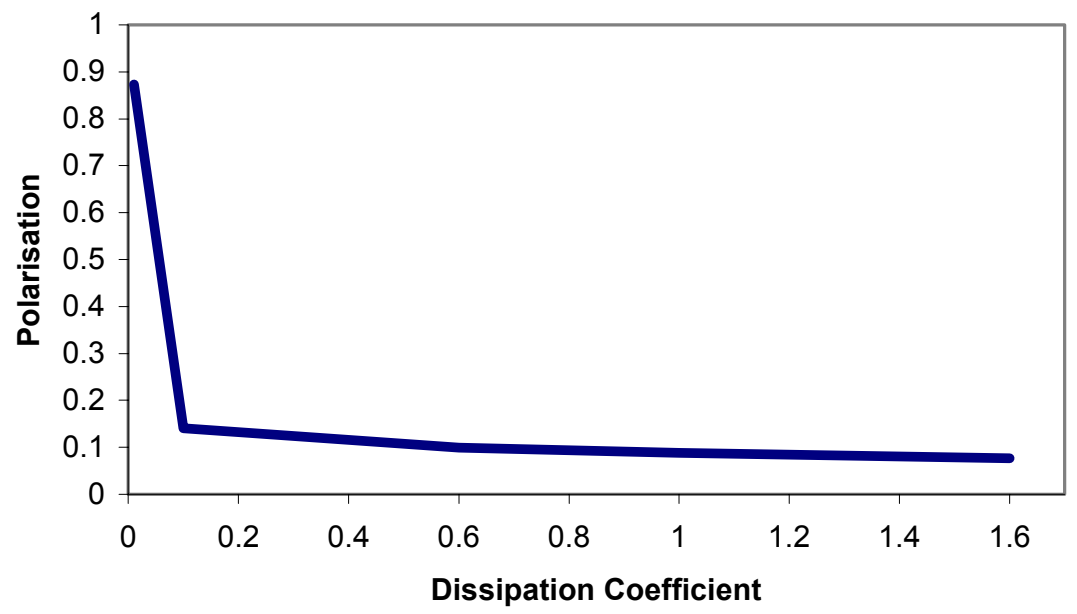

Fig.1. Effect of different dissipative coefficients on group polarization.

$$
\left(C_{a}=1 ; C_{l}=1.2 ; C_{0}=0.1 ; l_{a}=1 ; I_{l}=0.2 ; I_{0}=1.5 ; m=1\right)
$$


(a)

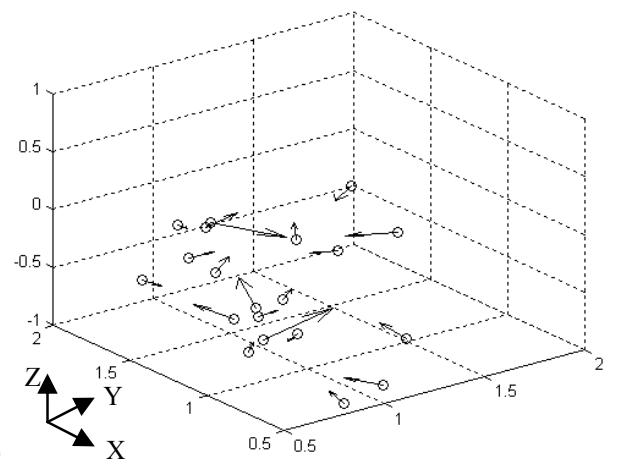

(b)

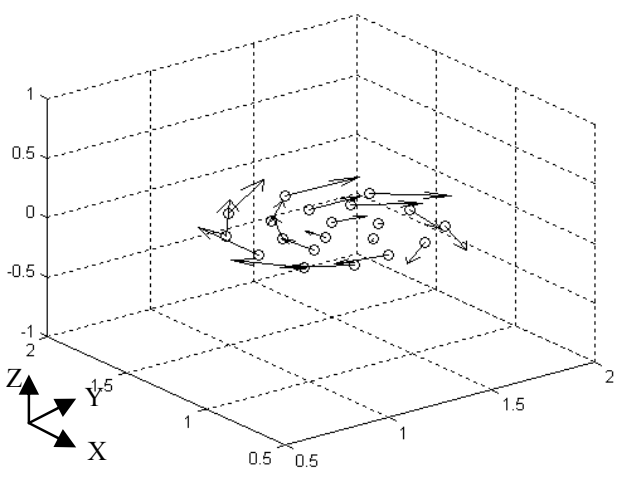

Fig.2. Simulation of the group motion according to the model with no dissipative forces. $C_{a}=1, C_{r}=1.2, C_{o}=0.1, I_{a}=1, I_{r}=0.2, I_{o}=1.5, m=1, N=20,(a) \mathrm{t}=0$ (b) $\mathrm{t}=14$

(a)

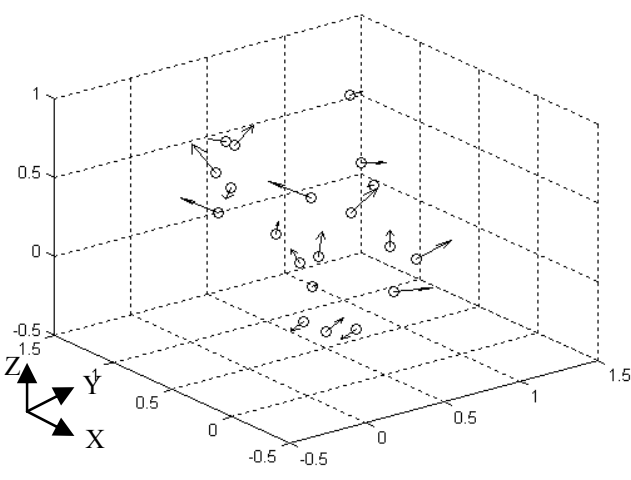

(b)

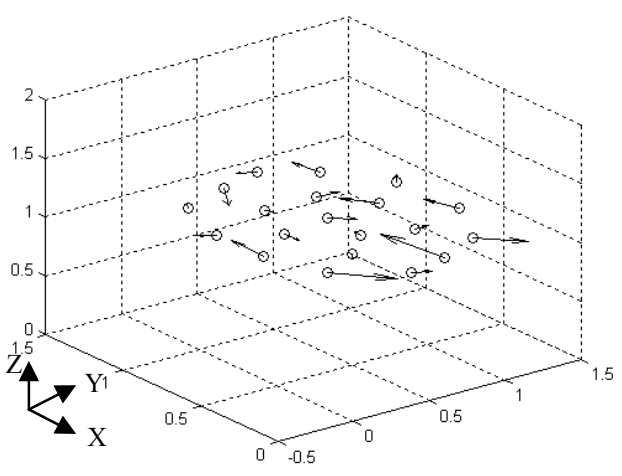

Fig.3. Simulation of the group motion according to the model with dissipative forces. $C_{a}=1, C_{r}=1.2, C_{o}=0.1, l_{a}=1, I_{r}=0.2, I_{o}=1.5, m=1, \beta=0.1, N=20,(a) t=0$ (b) $\mathrm{t}=14$ 


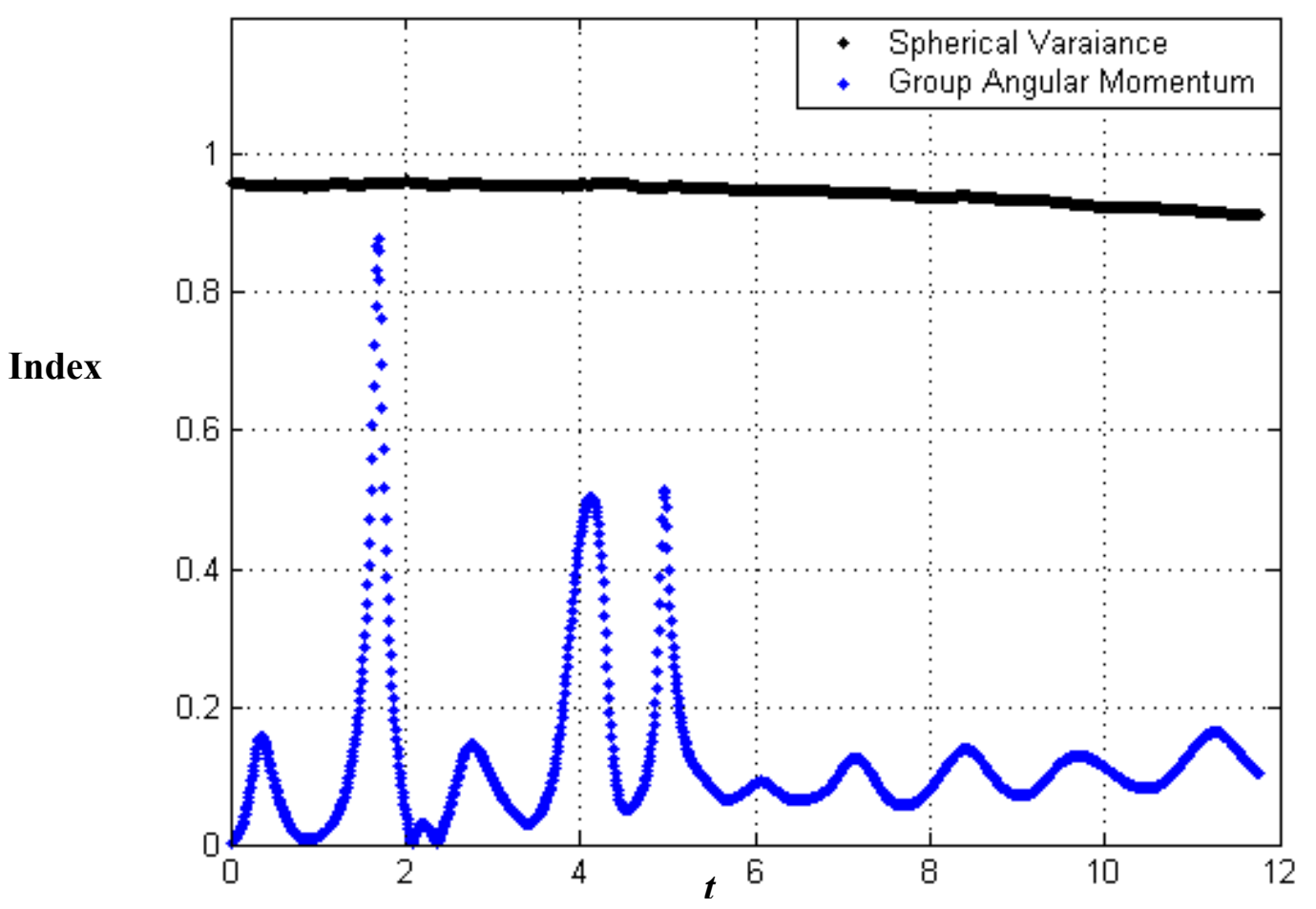

Fig.4. Group spherical variance and angular momentum versus time. $\left(C_{a}=1 ; C_{r}=1.2 ; C_{o}=0.1 ; l_{a}=1 ; I_{r}=0.2 ; I_{o}=1.5 ; m=1, \beta=0.01\right)$

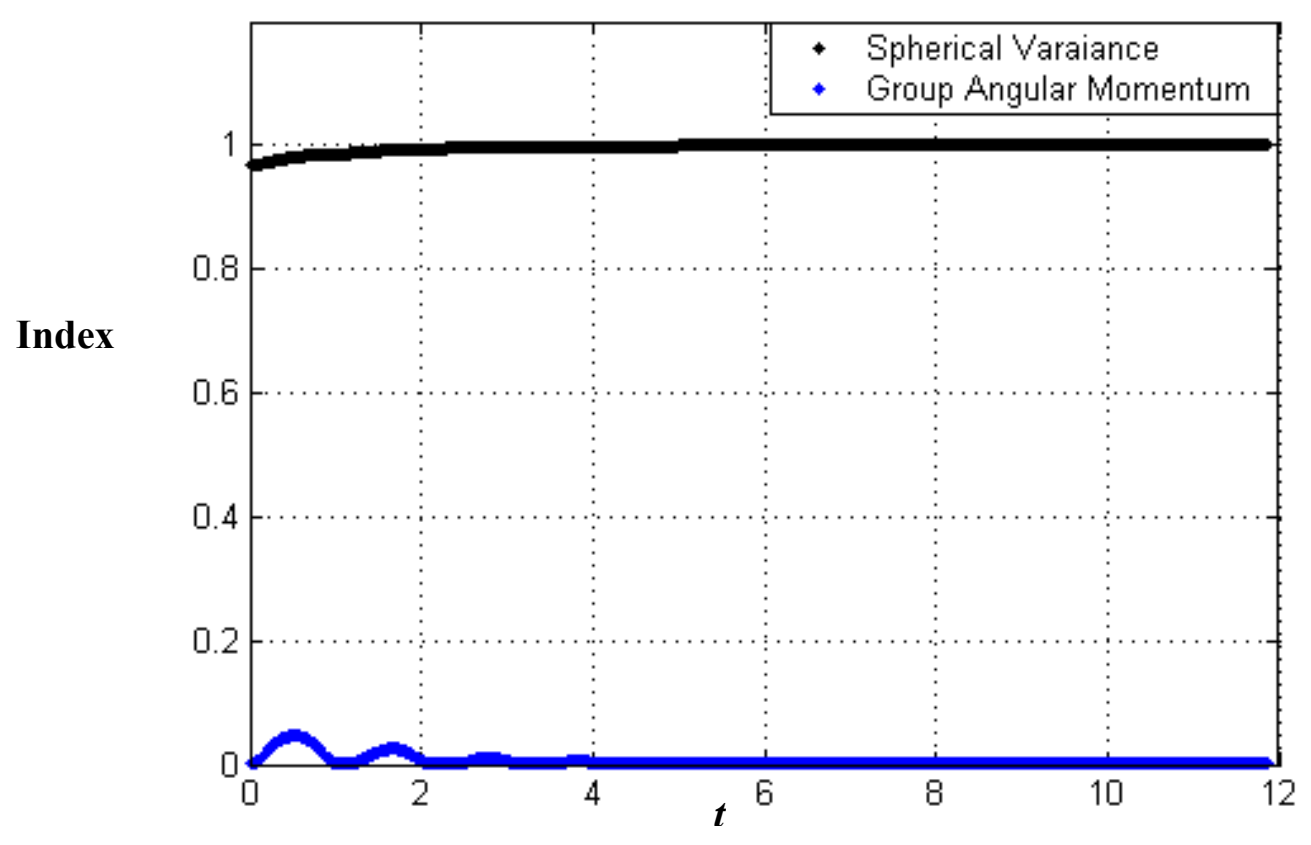

Fig.5. Group spherical variance and angular momentum versus time. $\left(C_{a}=1 ; C_{r}=1.2 ; C_{o}=0.1 ; I_{a}=1 ; I_{r}=0.2 ; I_{o}=1.5 ; m=1, \beta=1.8\right)$ 


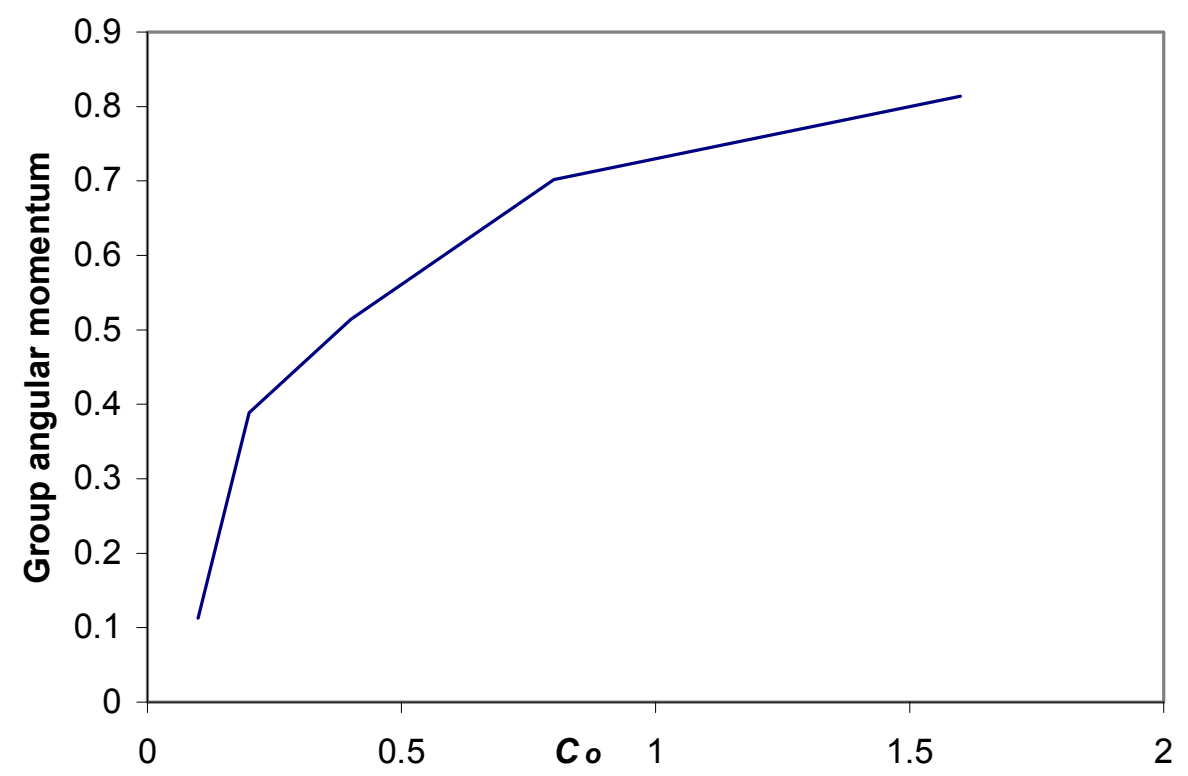

Fig.6. Group angular momentum as a function of $C_{0}$. $\left(I_{r}=0.2, I_{a}=1, C_{r}=1.2, C_{a}=1, \beta=0.1\right)$

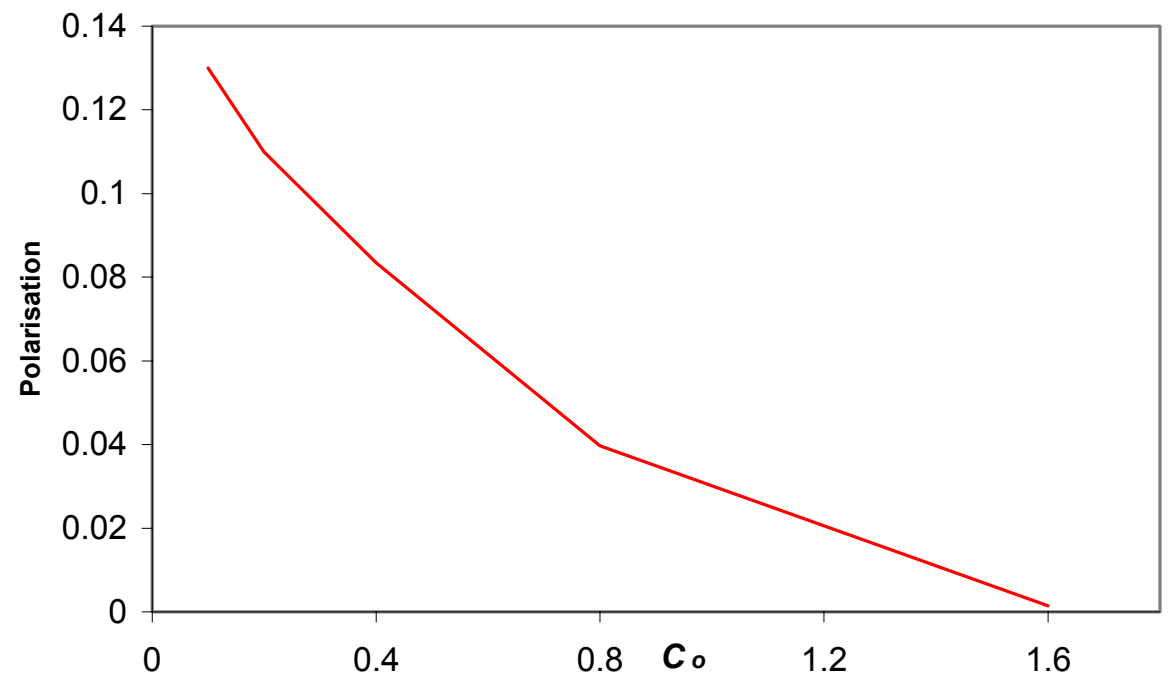

Fig.7. Group polarization as a function of $C_{0}$. $\left(I_{r}=0.2, I_{a}=1, C_{r}=1.2, C_{a}=1, \beta=0.1\right)$ 


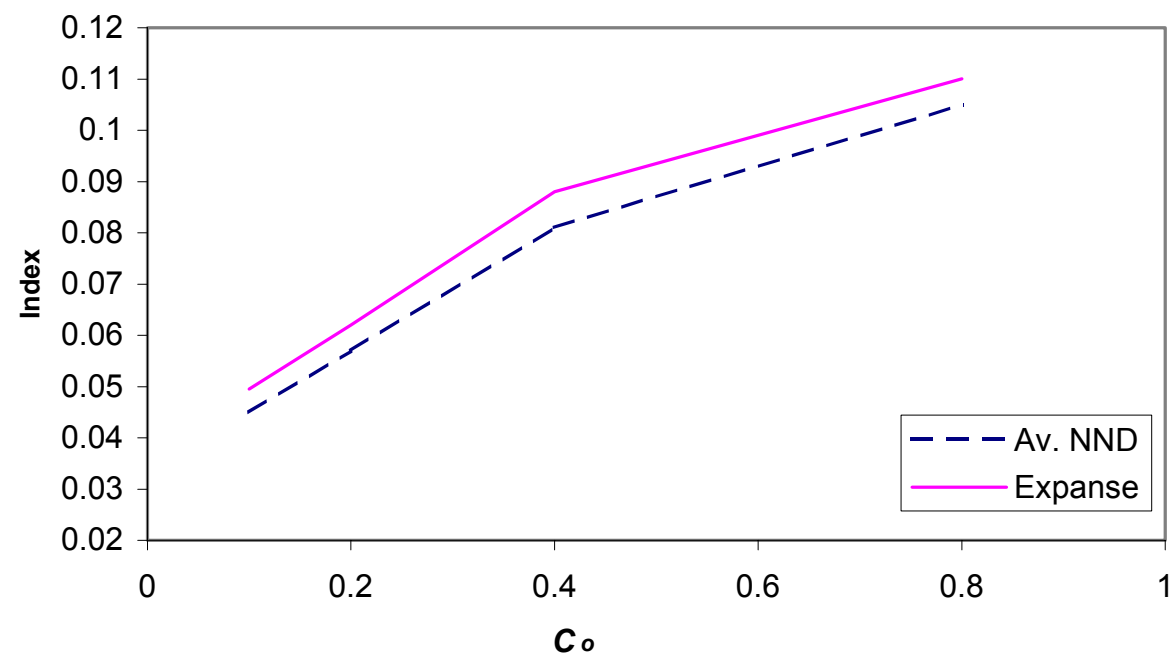

Fig.8. Group expanse and average NND as a function of $C_{0}$. $\left(I_{r}=0.2, I_{a}=1, C_{r}=1.2, C_{a}=1, \beta=0.1\right)$

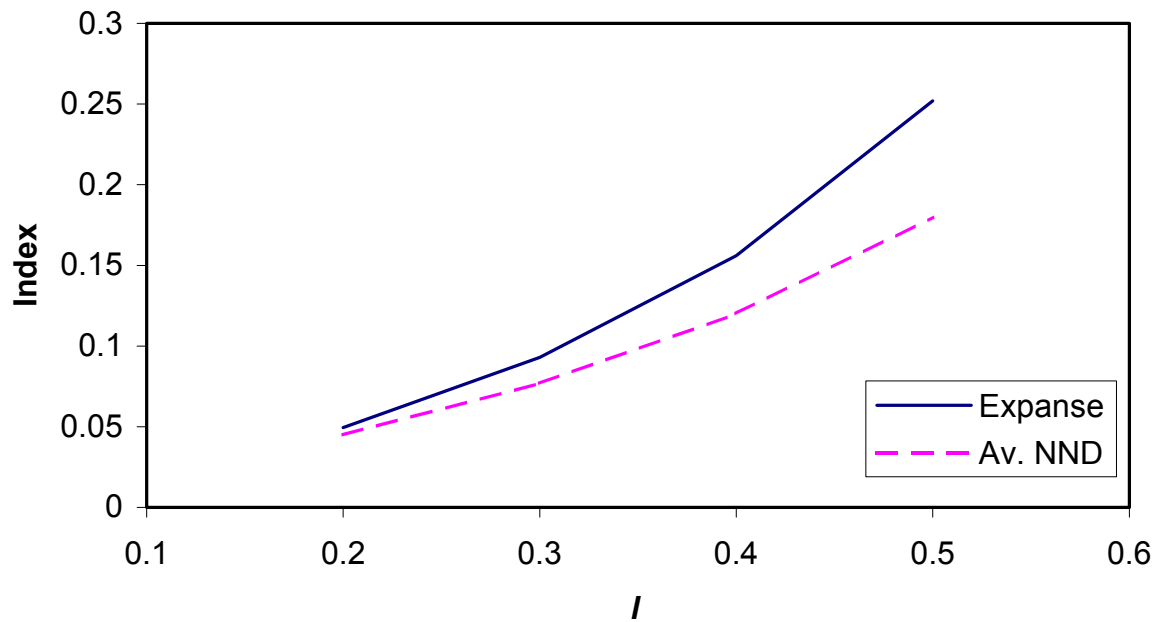

Fig.9. Group expanse and average NND as a function of the ratio $I\left(I_{r} I_{a}\right) .\left(C_{r}=1.2, C_{a}=1, \beta=0.1\right)$ 


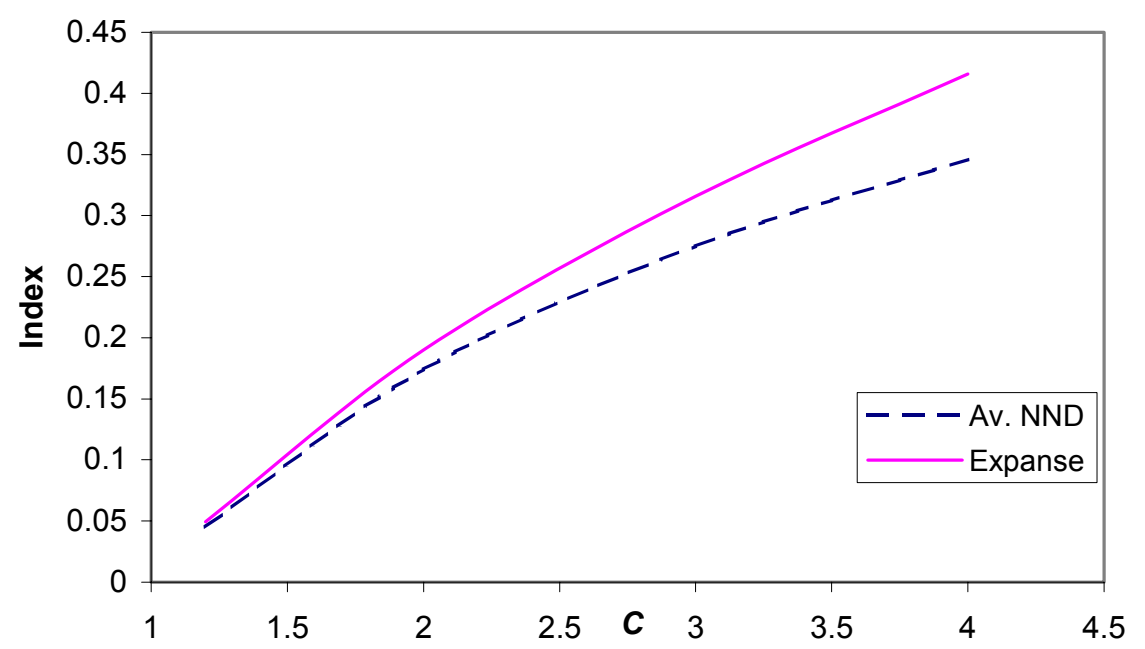

Fig.10. Group expanse and average NND as a function of the ratio $C\left(C_{r} / C_{a}\right) \cdot\left(I_{r}=0.2, l_{a}=1, \beta=0.1\right)$
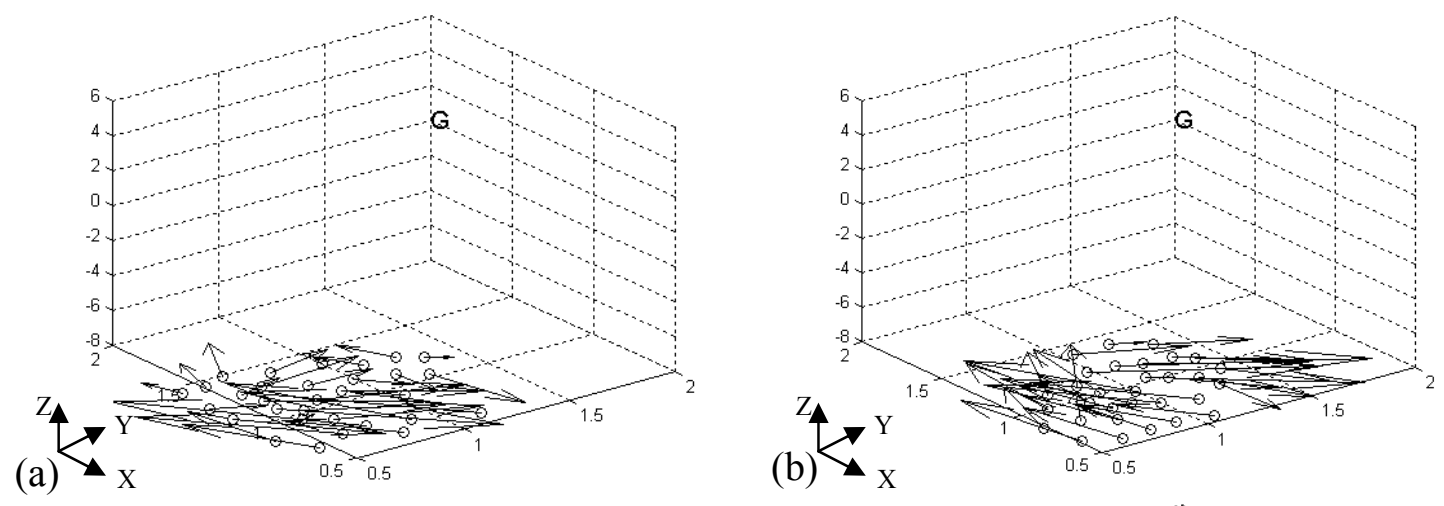

(c)
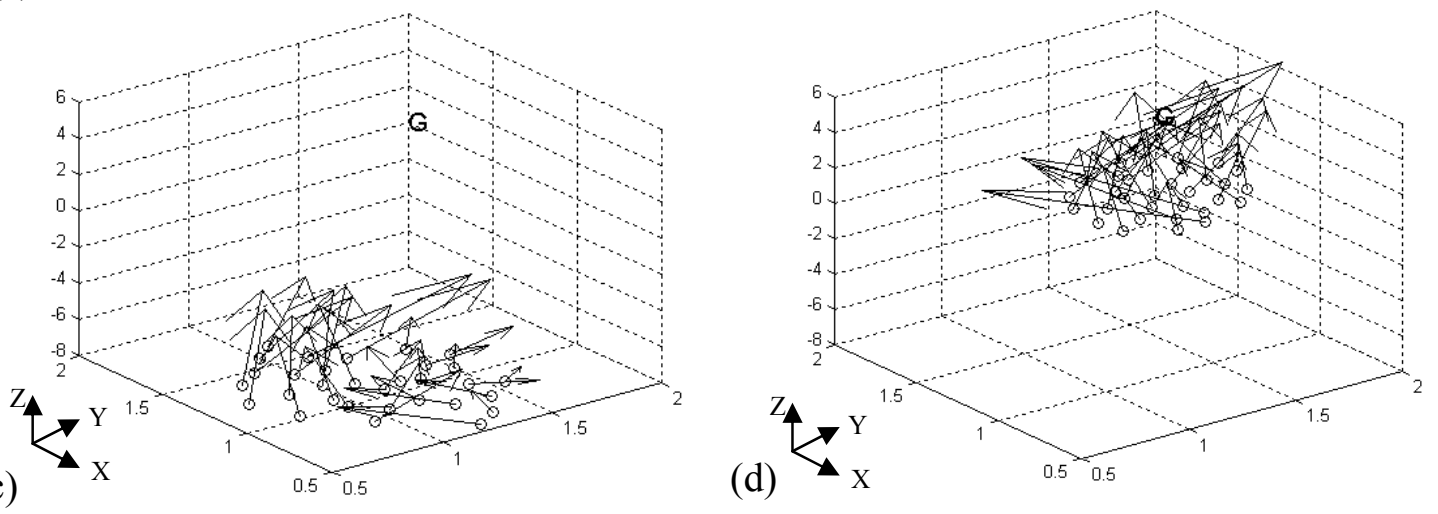

Fig.11. Simulation of the group motion in a vortex like shape pattern approaching a potential sink at point $G$. $C_{g}=15, C_{a}=1, C_{r}=1, C_{o}=0.7, I_{g}=5, l_{a}=1, I_{r}=0.2, I_{0}=1.5$, $m=1, \beta=1, N=31$, (a) $t=0$ (b) $t=28$ (c) $t=86$ (d) $t=318$ 